\title{
Primary anterior shoulder dislocation in young athletes: fix them!
}

\author{
Pietro Randelli $\cdot$ Ettore Taverna
}

Published online: 7 October 2009

(C) Springer-Verlag 2009

The shoulder is a versatile complex of joints with a wide functional range of motion and stability. Reports from both the general population and military population in the United States and Europe showed an annual incidence of 0.08-1.69 dislocations per 1,000 people [13]. The younger the population, the higher the risk of dislocation.

Historically, the first episode of anterior shoulder dislocation was treated non-surgically and surgical stabilization was considered first after a recurrence. This approach, useful in all ages, confirmed the effectiveness of a conservative protocol, with $52 \%$ of the shoulders never experiencing a redislocation [8].

Nowadays, non-surgical treatment, after the initial reduction of the gleno-humeral joint, includes a period of immobilization in a position of external rotation [9] followed by physical therapy for up to 3 months.

Even if it was postulated that the Bankart lesion has the ability to heal when immobilized in external rotation [9], few recent studies raised concerns. Finestone et al. [6] showed similar risk of recurrence in a prospective study comparing external rotation to internal rotation bracing after primary traumatic anterior dislocation. Limpisvasti et al. [12] asserted that external rotation does not create an adaptation of the Bankart lesion by the Subscapularis muscle. Scheibel et al. [14] used MRI to study the grade of adaptation of the labrum to the glenoid rim in different

P. Randelli ( $\square)$

Dipartimento di Scienze Medico-Chirurgiche,

Policlinico San Donato IRCCS, Università degli Studi di Milano,

Via Morandi 30, San Donato Milanese, 20097 Milan, Italy

e-mail: pietro.randelli@unimi.it

E. Taverna

Istituto Ortopedico Galeazzi, Milan, Italy positions of rotation. These researchers stated that the optimum position of immobilization in external rotation has not been determined yet.

However, this approach proved to be associated with a high rate of recurrence, particularly in young athletic population. The recurrence rate in active individuals younger than 25 years was reported to be between 17 and $96 \%$ [11].

Several studies, even with Level I evidence, compared the results of immediate surgical stabilization after the firsttime dislocation to those of non-operative treatments. Surgery was proved to reduce the recurrence rate after a first-time anterior shoulder dislocation. The recurrence rate in the group of conservatively treated patients ranged between 60 and $75 \%$. In surgically treated groups, the recurrence was between 11 and 20\% [2, 11]. Even in a long-term follow-up [10], surgical stabilization gave better results, with approximately $10 \%$ recurrence rate compared to $58 \%$ recurrence in the conservative group.

A cochrane review [7] as well as a recent systematic review by Brophy and Marx [4] suggested an early surgical repair of the Bankart lesion in young active male patients, especially if they are engaged in demanding physical activities.

Whether the surgical Bankart repair should be an open or arthroscopic, method is still a matter of debate. Recent studies showed no differences in terms of recurrences rate $[3,5]$ as well as shoulder scores. The only difference was that open Bankart repair gave a higher average surgical time [3].

The debate about the correct indication for Bankart repair is ongoing. The recurrence rate after arthroscopic Bankart repair in unselected patients suggested the necessity of a very careful patient selection. Balg and Boileau [1] identified recurrent instability as the main complication 
after arthroscopic Bankart repair. The recurrence rate ranged between 5 and $20 \%$.

The important question is whether there is a possibility to reduce the recurrence rate. First of all, we need to identify the risk factors. These are prognostic factors that identify patients who will develop recurrent instability after an arthroscopic Bankart procedure and who might be more effectively treated with a different procedure (i.e. a bony augmentation procedure like Latarjet-Bristow).

Boileau and Balg [1] identified the following risk factors: patients under 20 years of age at the time of surgery involved in competitive or contact sports or those involving forced overhead activity, shoulder hyperlaxity, a HillSachs lesion shown on the antero-posterior radiograph of the shoulder in external rotation, and/or loss of the sclerotic inferior glenoid contour. These factors were integrated in a 10-point pre-operative instability severity index score (ISI). The researchers suggested that patients with a score higher than six points had an unacceptable recurrence risk; i.e. approximately $70 \%$, and an open Latarjet procedure in these patients should therefore be preferred. Of course, the ISI score represents only a part of a puzzle that needs to be verified in prospective studies.

In conclusion, recent studies suggest an operative approach to the first-time anterior dislocation of the shoulder, especially if the patient is young, active, and involved in highly demanding sports. The choice of surgical procedure is between an arthroscopic suture anchors repair and a bony procedure, open or possibly arthroscopic.

The next 10 years will give us the answers.

\section{References}

1. Balg F, Boileau P (2007) The instability severity index score. A simple pre-operative score to select patients for arthroscopic or open shoulder stabilisation. J Bone Joint Surg Br 89:1470-1477

2. Bottoni CR, Wilckens JH, DeBerardino TM et al (2002) A prospective, randomized evaluation of arthroscopic stabilization versus nonoperative treatment in patients with acute, traumatic, first-time shoulder dislocations. Am J Sports Med 30:576-580
3. Bottoni CR, Smith EL, Berkowitz MJ, Towle RB, Moore JH (2006) Arthroscopic versus open shoulder stabilization for recurrent anterior instability: a prospective randomized clinical trial. Am J Sports Med 34:1730-1737

4. Brophy RH, Marx RG (2009) The treatment of traumatic anterior instability of the shoulder: nonoperative and surgical treatment. Arthroscopy 25:298-304

5. Fabbriciani C, Milano G, Demontis A, Fadda S, Ziranu F, Mulas PD (2004) Arthroscopic versus open treatment of Bankart lesion of the shoulder: a prospective randomized study. Arthroscopy $20: 456-462$

6. Finestone A, Milgrom C, Radeva-Petrova DR, Rath E, Barchilon V, Beyth S, Jaber S, Safran O (2009) Bracing in external rotation for traumatic anterior dislocation of the shoulder. J Bone Joint Surg Br 91:918-921

7. Handoll HHG, Almaiyah MA (2004) Surgical versus non-surgical treatment for acute anterior shoulder dislocation. Cochrane Database Syst Rev 1:CD004325

8. Hovelius L, Augustini BG, Fredin H, Johansson O, Norlin R, Thorling J (1996) Primary anterior dislocation of the shoulder in young patients. A ten-year prospective study. J Bone Joint Surg Am 78:1677-1684

9. Itoi E, Hatakeyama Y, Sato T, Kido T, Minagawa H, Yamamoto N, Wakabayashi I, Nozaka K (2007) Immobilization in external rotation after shoulder dislocation reduces the risk of recurrence. A randomized controlled trial. J Bone Joint Surg Am 89:21242131

10. Jakobsen BW, Johannsen HV, Suder P, Sojbjerg JO (2007) Primary repair versus conservative treatment of first-time traumatic anterior dislocation of the shoulder: a randomized study with 10-year follow-up. Arthroscopy 23:118-123

11. Kirkley A, Werstine R, Ratjek A, Griffin S (2005) Prospective randomized clinical trial comparing the effectiveness of immediate arthroscopic stabilization versus immobilization and rehabilitation in first traumatic anterior dislocations of the shoulder: long-term evaluation. Arthroscopy 21:55-63

12. Limpisvasti O, Yang BY, Hosseinzadeh P, Leba TB, Tibone JE, Lee TQ (2008) The effect of glenohumeral position on the shoulder after traumatic anterior dislocation. Am J Sports Med 36:775-780

13. Owens BD, Dawson L, Burks R, Cameron KL (2009) Incidence of shoulder dislocation in the United States Military: demographic considerations from a high-risk population. J Bone Joint Surg Am 91:791-796

14. Scheibel M, Kuke A, Nikulka C, Magosch P, Ziesler O, Schroeder RJ (2009) How long should acute anterior dislocations of the shoulder be immobilized in external rotation? Am J Sports Med 37:1309-1316 